\title{
Routine Probiotic Use in Very Preterm Infants: Retrospective Comparison of Two Cohorts
}

\author{
Francesco Bonsante, MD 1,2,3 Silvia lacobelli, MD 1,2,3 Jean-Bernard Gouyon, MD, PhD 1,3,4
}

${ }^{1}$ NICU, Department of Paediatrics, University of Dijon, France

${ }^{2}$ Neonatology and NICU, GHSR, CHR, Saint Pierre Réunion, France

${ }^{3}$ Centre d'Etudes Périnatales de l'Océan Indien, Centre d'Investigation Clinique et d'Epidémiologie Clinique (CIC-EC) CHR, Réunion, France

${ }^{4}$ Centre d'Epidémiologie des Populations (EA4184), Université de

Dijon, Dijon, France

\begin{abstract}
Address for correspondence and reprint requests Francesco Bonsante, MD, Neonatology and NICU, CHR, BP350 97448 Saint Pierre Cedex, France (e-mail: fbonsante@yahoo.com).
\end{abstract}

\begin{abstract}
Keywords

- VLBWI

- preterm birth

- probiotics

- NEC

- enteral nutrition

- NICU

- Lactobacillus casei rhamnosus 35 (Lcr Restituo)

Objective Evidence supports the efficacy of probiotics in reducing necrotizing enterocolitis (NEC) in very low-birth-weight infants, although concerns remain with regard to their routine use. Since 2008 in our neonatal intensive care unit, a low dose of probiotics (unique strain) is administered as standard of care in all preterm babies born at 24 to 31 weeks' gestation. This study reports outcomes in infants receiving probiotic cohort (PC) compared with the historical cohort.

Design Treatment with Lactobacillus rhamnosus Lcr35 (Lcr Restituo) $\left(2 \times 10^{8}\right.$ colonyforming units $/ 12 \mathrm{~h}$ ) was started early after birth and intention to treat was up to 36 weeks' gestation. The main outcome was definite NEC. Secondary outcomes were mortality, late-onset sepsis (LOS), cholestasis, isolated rectal bleeding (IRB), and time to reach full enteral feeding (FEF).

Results A total of 1130 patients were included. No adverse effects were observed. Infants in PC presented a reduced rate of NEC (odds ratio [OR] 0.20; 95\% confidence interval $[\mathrm{Cl}] 0.07$ to 0.58 ), mortality (OR $0.46 ; 95 \% \mathrm{Cl} 0.21$ to 1.00 ), and LOS (OR 0.60; $95 \% \mathrm{Cl} 0.40$ to 0.89 ) and achieved FEF significantly earlier. IRB was significantly reduced among infants receiving the complete scheduled treatment.

Conclusion Administration of $L$ cr Restituo was well tolerated and associated with lower mortality and morbidities in this cohort. Our results provide evidence in support of the hypothesis that this probiotic may reduce IRB.
\end{abstract}

The use of probiotics for preventing necrotizing enterocolitis (NEC) in very low birth weight infants (VLBWI) is currently a "hot topic" in neonatal literature, due to some controversial and open issues in the scientific debate. There is definitely an increasing body of evidence that probiotics represent an important tool in reducing the risk of severe NEC in premature babies ${ }^{1,2}$; however, some caution about encouraging their widespread use has been suggested. ${ }^{3,4}$ For instance, the level of evidence for routine use of probiotics is at present considered inadequate in the area of product, dosage, treatment modalities, and longterm effects, and the need for more research has been recently contended by international experts and committees. ${ }^{5-9}$

Probiotics are widely used in Europe and some neonatal intensive care units (NICUs) in Italy and Finland have been using them routinely for several years, without reporting any significant adverse effect. ${ }^{10,11}$ Recently, Denmark has issued some guidelines for the use of probiotics in preterm newborns. ${ }^{12}$ received

December 1, 2011

accepted after revision

March 21, 2012

published online

July 6, 2012
Copyright $\odot 2013$ by Thieme Medical Publishers, Inc., 333 Seventh Avenue, New York, NY 10001, USA. Tel: +1(212) 584-4662.
DOI http://dx.doi.org/ 10.1055/s-0032-1321498. ISSN 0735-1631. 
In view of the growing evidence for the beneficial effects of probiotics in reducing all-cause mortality and definite NEC in VLBWI, in July 2008 we introduced the administration of Lactobacillus casei rhamnosus, Lcr35 (Lcr Restituo) as a standard of care in all preterm infants born at less than 32 weeks' gestation and hospitalized in the NICU of Dijon university hospital (France).

The aim of this study was to report the neonatal outcomes of preterm infants admitted to our NICU and receiving routine administration of the probiotic Lcr Restituo since birth, by comparing them with a retrospective, historical cohort.

\section{Methods}

\section{Design}

The study design was an observational retrospective cohort analysis.

\section{Setting and Years of the Study}

The study was conducted in the tertiary NICU of Dijon university hospital during two consecutive periods: from January 1 , 2003, to June 30, 2008 (historical cohort [HC]) and from July 1, 2008, to April 30, 2011 (probiotic cohort [PC]).

\section{Study Population}

All the infants born between 24 and 31 weeks of gestational age (GA) and hospitalized in the NICU within 48 hours after birth were eligible for the analysis. Patients were excluded if clinical data about hospital stay, neonatal outcomes, and adverse events were not available or were incomplete, if they did not receive any enteral feeding during the first 48 hours of life or if they presented congenital gastrointestinal malformations. A criterion for secondary exclusion was death within the first week of life.

\section{Probiotic Administration Guidelines and Enteral Nutrition Practice in the NICU}

Infants in the PC received two daily administrations of $2 \times 10^{8}$ cells per unit of a unique strain of Lcr Restituo. The probiotic formulation was prepared and stored in closed capsules by the manufacturer (Probionov, France) and purchased by the hospital pharmacy. Capsules were opened and mixed with $1 \mathrm{~mL}$ of sterile water immediately before administration to infants by the gastric feeding tube.

Probiotic supplementation was started as soon as minimal enteral feeding was commenced. Probiotic supplementation was maintained in cases of clinical instability (for example proven or suspected sepsis, respiratory distress, or patent ductus arteriosus) if any enteral feeding was maintained, and it was discontinued whenever enteral feeding was interrupted.

Treatment schedule dictated that supplementation would stop at the corrected GA of 36 weeks or at the hospital discharge.

During the two study periods, there were no major changes in the unit nutritional practice, with the exception that in November 2006, a standardized parenteral nutrition (SPN) regimen was introduced in our NICU for routine use in substitution of the individualized parenteral nutrition (IPN), as already described. ${ }^{13}$ In both regimens, parenteral nutrition was started as soon as possible after birth. Exclusive breast milk (mother or donor banked) was used in the NICU and according to what was suggested, ${ }^{14}$ all very preterm infants in the unit benefited from a standardized feeding schedule.

Minimal enteral feeding was started within the first hours after birth and continued for 2 to 6 days depending on the infant GA; after this, oral intake was increased, if tolerated, by $20 \mathrm{~mL} / \mathrm{kg} / \mathrm{d}$. Human milk fortification was started when enteral feeding achieved intakes of 130 to $140 \mathrm{~mL} / \mathrm{kg} / \mathrm{d}$, and parenteral nutrition was discontinued at the same time.

During the study period, there were no main changes in the NICU policies and protocols possibly related with the onset of NEC.

\section{Data Collection}

In Burgundy perinatal services are regionalized with three levels of care, and our unit is the only III level NICU in the region. Clinical data on the newborn population are collected by the regional perinatal database of the Burgundy perinatal network. ${ }^{15,16}$ This database was set up with the approval of the National Committee of Informatics and Liberty and prospectively records clinical events for mothers and infants between birth and hospital discharge. For the purpose of this work, information was retrieved from the regional database and from the medical files.

The following data were collected: use of antenatal steroids, mode of delivery, GA, birth weight, gender, Apgar score at 1 minute, use of antibiotics and proton pump inhibitors, and kind of parenteral nutrition (SPN or IPN).

\section{Outcome Measures}

The main outcome chosen for the analysis was definite NEC (NEC stage II or higher) according to Bell's classification.

The secondary outcomes were: all stages NEC, mortality (death after 7 days of life), proven late-onset sepsis (LOS), treated cholestasis, isolated rectal bleeding (IRB), time to reach full enteral feeding, overall hospital stay, and NICU length of stay.

The time of the event was recorded for definite NEC, all stages NEC, LOS, and IRB.

The following adverse effects were recorded: proven sepsis attributed to Lactobacillus sp., abdominal distension that required enteral feeding to be stopped (for more than 12 hours), vomiting (at least 2 episodes in 24 hours), and diarrhea (at least two episodes in 24 hours).

\section{Laboratory Methods}

Microbiological cultures for suspected LOS were realized when indicated by the attending physician and clinically justified.

Microbiology laboratory was able to culture the probiotic on appropriate culture media.

\section{Statistical Analysis}

Data are presented as mean and standard deviation for continuous variables and as numbers and proportions (\%) 
for categorical ones. Comparisons between groups were performed by using $\mathrm{X}^{2}$ test or Fisher exact test for categorical variables; odds ratio with $95 \%$ confidence interval was also calculated. To estimate the independent influence of probiotic on the main outcome, we have realized a logistic multiple regression model using three predictor variables-probiotic and two potentially confounding baseline variables.

Analysis of variance test was used for parametric variables and the Mann-Whitney $U$ test for nonparametric continuous variables; $p$ values $<0.05$ were considered statistically significant. All statistical analyses were performed using SPSS (SPSS for Windows, version 16.0, 2008, Chicago, IL).

\section{Ethics and Consent}

Due to the retrospective character of this study, approval of the research ethics committee in our hospital was not needed. According to French legislation, written parental consent was not needed for this retrospective study.

\section{Results}

From January 1, 2003, to April 30, 2011, 1206 neonates born before 32 weeks of gestation were hospitalized in our NICU. Clinical data and neonatal outcomes were available for all of them. Of these, 1189 were admitted within hour 48 of life. Among them, 29 were excluded: six due to congenital gastrointestinal abnormalities and 23 because they had not received any enteral feeding during the first 48 hours of life. Thirty infants were secondarily excluded because death occurred within 7 days of life (19 in the HC and 11 in the PC). Excluded patients were equally distributed between the two groups. Finally, 1130 infants were considered for the analysis, 783 in the HC and 347 in the PC.

- Table 1 shows demographic and clinical data of the two groups.

In the PC, treatment was begun during the first day of life in $85.5 \%$ of infants. The median age at the outset was 16 hours of life (6 to 36 hours). Two hundred ninety-five infants (85\% percent of those treated) received a complete course of probi- otic until 36 weeks of corrected GA or until hospital discharge. For the other 52 infants, treatment was stopped at the time of the transfer from the NICU to a secondary care unit. In this subgroup, probiotic administration was stopped at a median corrected GA of 32 weeks and 3 days; there was no difference for clinical characteristics when compared with infants receiving a complete course of probiotics (data not shown).

- Table 2 shows the results of main and secondary outcomes. Infants in the PC had a significantly lower rate of NEC, mortality, LOS, and NICU length of stay when compared with infants in the HC. Time to reach full enteral feeding was significantly shorter in the PC.

The effect of probiotics on the main outcome remained statistically significant when adjusted for confounding baseline variables (use of antibiotics and SPN).

When the analysis was limited to the infants receiving a complete course of probiotic treatment, the efficiency was further enhanced for each of the outcomes (data not shown), and Lcr Restituo was significantly helpful in reducing the rate of IRB ( $p=0.04$ versus the HC).

The occurrence of LOS was significantly delayed in the PC compared with the HC (data shown on - Table $\mathbf{3}$ ).

Finally, the expected adverse effects were similar between groups (-Table 4).

\section{Discussion}

Our report shows that the prophylactic administration of $\mathrm{Lcr}$ Restituo is associated with a reduced risk of severe NEC and late mortality without significant adverse effects in the treated population when compared with a historical cohort. This is consistent with the results of several randomized controlled trials demonstrating the beneficial effects of probiotics. ${ }^{17-20}$ Nowadays a very lively debate is taking place on the advisability of the routine use of probiotics in preterm infants, 7,21 and it involves many topics. First, what is the power of evidence for recommending probiotics as a standard of care for premature babies? ${ }^{22}$ Second is how to deal with all the practical aspects of probiotic supplementation, ${ }^{23}$

Table 1 Characteristics of the Study Population

\begin{tabular}{|l|l|l|l|}
\hline & Probiotic Cohort $(\boldsymbol{n}=347)$ & Historical Cohort $(\boldsymbol{n}=\mathbf{7 8 3})$ & $\boldsymbol{p}$ \\
\hline Gestational age (wk) & $28.9 \pm 1.9$ & $28.9 \pm 1.8$ & n.s. \\
\hline Gestational age <28 wk (\%) & 29 & 25 & n.s. \\
\hline Birth weight (g) & $1220 \pm 353$ & $1214 \pm 329$ & n.s. \\
\hline Sex male (\%) & 51 & 57 & n.s. \\
\hline Antenatal steroids (\%) & 83 & 84 & n.s. \\
\hline Apgar score <3 at 1 min (\%) & 8.0 & n.s. \\
\hline Standardized parenteral nutrition (\%) & 100 & 46 & 0.0000 \\
\hline Antibiotics in the first 96 h of life (\%) & 77 & 31 & 0.04 \\
\hline Proton pump inhibitors (\%) & 32 & & n.s. \\
\hline
\end{tabular}

Study population: 1130 infants born at 24-31 wk of gestation. Continuous data are expressed as mean and standard deviation, and calculations were performed with analysis of variance test. Categorical data are expressed as percentage, and calculations were performed with $\mathrm{x}^{2}$ test. $\mathrm{n}$.s., not significant. 
44 Probiotics and Very Preterm Infants Bonsante et al.

Table 2 Outcomes of the Study Population

\begin{tabular}{|l|l|l|l|l|}
\hline & $\begin{array}{l}\text { Probiotic Cohort } \\
(n=347)\end{array}$ & $\begin{array}{l}\text { Historical Cohort } \\
(\boldsymbol{n}=783)\end{array}$ & OR (95\% CI) \\
\hline NEC stage 2-3 (\%) & 1.2 & 5.3 & $0.20(0.07-0.58)$ & 0.0009 \\
\hline NEC stage 2-3 adjusted (\%) & 1.2 & 5.3 & $0.23(0.08-0.69)$ & 0.008 \\
\hline $\begin{array}{l}\text { NEC stage 2-3 in infants less than } \\
\text { 28 wk of gestation (\%) }\end{array}$ & 1.0 & 10.6 & $0.08(0.01-0.63)$ & 0.002 \\
\hline NEC all stages (\%) & 2.9 & 7.9 & $0.34(0.17-0.68)$ & 0.001 \\
\hline Mortality (\%) & 2.3 & 4.8 & $0.46(0.21-1.00)$ & 0.04 \\
\hline Isolated rectal bleeding (\%) & 4.6 & 7.5 & $0.59(0.34-1.05)$ & 0.07 \\
\hline Late-onset sepsis (\%) & 10.7 & 16.6 & $0.60(0.40-0.89)$ & 0.01 \\
\hline Cholestasis (\%) & 3.5 & 4.3 & $0.79(0.40-1.55)$ & n.s. \\
\hline Full enteral feeding (day of life) & $11.7 \pm 10.0$ & $16.5 \pm 13.3$ & & 0.0005 \\
\hline NICU discharge (day of life) & $26.7 \pm 26.3$ & $30.3 \pm 28.0$ & $68.8 \pm 28.4$ & \\
\hline Hospital discharge (day of life) & $73.8 \pm 39.1$ & 0.03 \\
\hline
\end{tabular}

Categorical data are expressed as percentage, and calculations were performed with $x^{2}$ test or Fisher exact test. Continuous data are expressed as mean and standard deviation, and calculation were performed with Mann-Whitney $U$ test. Cl, confidence interval; NEC, necrotizing enterocolitis; NICU, neonatal intensive care unit; n.s., not significant; OR, odds ratio.

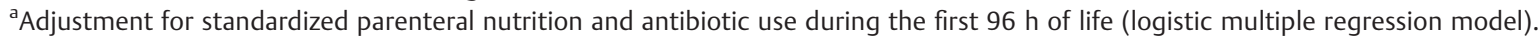

Table 3 Time of Event (Day of Life) in the Study Population

\begin{tabular}{|l|l|l|l|}
\hline & Probiotic Cohort $(\boldsymbol{n}=347)$ & Historical Cohort $(\boldsymbol{n}=783)$ & $\boldsymbol{p}$ \\
\hline NEC stage 2-3 & $30 \pm 1.7$ & $29 \pm 23$ & n.s. \\
\hline NEC all stages & $26 \pm 15$ & $29 \pm 22$ & n.s \\
\hline Sepsis & $25 \pm 14$ & $13 \pm 5.0$ & 0.03 \\
\hline Isolated rectal bleeding & $47 \pm 25$ & $37 \pm 28$ & 0.05 \\
\hline
\end{tabular}

Data are expressed as mean and standard deviation. Calculations are performed with analysis of variance test or Mann-Whitney U test. NEC, necrotizing enterocolitis; NICU, neonatal intensive care unit; n.s., not significant.

particularly those concerning strain and preparation to be used, adequate dosage, administration time, role of environmental cross-contamination, and the efficiency of probiotics in some specific infant populations (i.e., preterm babies exclusively fed mother or donor breast milk, rather than formula). ${ }^{24}$ Finally, more research is needed on the longterm effects of probiotics. ${ }^{9,24}$
We believe that, within the limits of some of the previous points, our work may provide additional information to that already reported by previous investigations.

As previously reported, ${ }^{25,26}$ probiotic use appears to facilitate enteral nutrition progression and achievement of full enteral feeding, and this result is particularly reliable considering that all the infants in this study only received human

Table 4 Adverse Effects in the Study Population

\begin{tabular}{|l|l|l|l|l|}
\hline & $\begin{array}{l}\text { Probiotic Cohort } \\
(n=347)\end{array}$ & $\begin{array}{l}\text { Historical Cohort } \\
(n=783)\end{array}$ & OR (95\% CI) \\
\hline Proven sepsis to Lactobacillus sp. (\%) & 0 & 0 & - & - \\
\hline $\begin{array}{l}\text { Stop enteral feeding for } \\
\text { abdominal distension (\%) }\end{array}$ & 2.9 & 2.9 & $0.98(0.46-2.09)$ & n.s. \\
\hline Diarrhea (\%) & 0.9 & 1.8 & $0.48(0.14-1.68)$ & n.s. \\
\hline Vomiting (\%) & 2.6 & 1.8 & $1.46(0.43-3.42)$ & n.s. \\
\hline Stop enteral feeding for any reason (\%) & 9.5 & 16.2 & $0.54(0.36-0.81)$ & 0.003 \\
\hline
\end{tabular}

Data are expressed as percentage; calculations were performed with $\mathrm{x}^{2}$ test or Fisher exact test. OR, odds ratio; $\mathrm{Cl}$, confidence interval; n.s., not significant. 
milk, and all along the study period, enteral nutrition guidelines did not change in our unit.

Other nuances of our results are of clinical interest: first, probiotic treatment was begun as soon as possible after birth in our population and earlier than in the earliest reported age at the start ${ }^{27}$; second, the supplementation was efficient despite using a unique strain and a dose smaller than reported in previous trials ${ }^{23}$; and finally, the duration of probiotic administration (until 36 weeks' gestation) could be important to optimize the protective effect of Lcr Restituo, especially with regard to the probiotic capacity of reducing the IRB. This outcome has never been assessed in previous clinical trials. Immunologic, allergic, or infectious mechanisms have been advocated in the pathogenesis of this often benign phenomenon appearing late during hospital stay in preterm babies, ${ }^{28}$ and some authors have evaluated the administration of Lactobacillus casei sp Rhamnosus GG (LGG) for improving hematochezia among infants with presumptive allergic colitis with beneficial effects. ${ }^{29}$ Other studies have documented that LGG reduces enteric pathogen colonization in premature infants ${ }^{30}$ and that some Lactobacillus spp. enhance the human intestinal barrier by modulating the local immune response. $^{31}$

Our study provides evidence in support of the hypothesis that this probiotic reduces IRB. The association reported did not reach statistical significance in the prespecified group of all infants of the PC, but it was significant in the subgroup of infants treated until 36 weeks of corrected age. This reflects perhaps a lack of statistical power. Thus, it would be interesting to further investigate and address the mechanisms for the eventual protective effect of Lcr Restituo on IRB of the preterm baby.

We acknowledge that the present study has several limitations, and the major one is undoubtedly its retrospective design. In addition, strict similarities between the two study periods with regard to clinical management and hospital infectious environment cannot be firmly ascertained. Moreover, the lack of a prospective and systematic surveillance of the microbiologic tolerability of the Lcr Restituo in our population is also regrettable and means that we cannot draw any conclusion about the overall safety of this probiotic in VLBWI. Finally, at the time of the introduction of probiotics in our NICU, no product was available in France for clinical use in newborns with the exception of $L C R$ Restituo, and we recognize that the use of a different strain than any of the previous studies represents a further limitation of our report.

Despite these limitations, the information reported about the efficacy of a low-dose, unique strain of probiotics in a cohort study remains valid. This finding further raises interest in addressing the previously mentioned issues regarding safety and efficiency in the future and prospective investigations on very preterm babies, thus representing a useful contribution to the comprehensive evaluation of probiotics' role in minimizing mortality and morbidity for this vulnerable population.

\section{References}

1 Alfaleh K, Anabrees J, Bassler D, Al-Kharfi T. Probiotics for prevention of necrotizing enterocolitis in preterm infants. Cochrane Database Syst Rev 2011;(3):CDOO5496

2 Deshpande G, Rao S, Patole S, Bulsara M. Updated meta-analysis of probiotics for preventing necrotizing enterocolitis in preterm neonates. Pediatrics 2010;125:921-930

3 Mihatsch WA, Braegger CP, Decsi T, et al. Critical systematic review of the level of evidence for routine use of probiotics for reduction of mortality and prevention of necrotizing enterocolitis and sepsis in preterm infants. Clin Nutr 2012;31:6-15

4 Neu J. Routine probiotics for premature infants: let's be careful!. J Pediatr 2011;158:672-674

5 Braegger C, Chmielewska A, Decsi T, et al; ESPGHAN Committee on Nutrition. Supplementation of infant formula with probiotics and/ or prebiotics: a systematic review and comment by the ESPGHAN committee on nutrition. J Pediatr Gastroenterol Nutr 2011;52: 238-250

6 Thomas DW, Greer FR; American Academy of Pediatrics Committee on Nutrition; American Academy of Pediatrics Section on Gastroenterology, Hepatology, and Nutrition. Probiotics and prebiotics in pediatrics. Pediatrics 2010;126:1217-1231

7 Soll RF. Probiotics: are we ready for routine use? Pediatrics 2010;125:1071-1072

8 Manzoni P, Rizzollo S, Vain N, et al. Probiotics use in preterm neonates: what further evidence is needed? Early Hum Dev 2011;87(Suppl 1):S3-S4

9 Neu J. Perinatal and neonatal manipulation of the intestinal microbiome: a note of caution. Nutr Rev 2007;65(6 Pt 1):282-285

10 Manzoni P, Lista G, Gallo E, et al. Routine Lactobacillus rhamnosus GG administration in VLBW infants: a retrospective, 6-year cohort study. Early Hum Dev 2011;87(Suppl 1):S35-S38

11 Luoto R, Isolauri E, Lehtonen L. Safety of Lactobacillus GG probiotic in infants with very low birth weight: twelve years of experience. Clin Infect Dis 2010;50:1327-1328

12 Danish National Guideline. Probiotics for preterm GA 30 weeks [in Danish]. Available at: http://www.rh-vejledninger.dk. Accessed November 2011

13 Iacobelli S, Bonsante F, Vintéjoux A, Gouyon JB. Standardized parenteral nutrition in preterm infants: early impact on fluid and electrolyte balance. Neonatology 2010;98:84-90

14 Patole SK, de Klerk N. Impact of standardised feeding regimens on incidence of neonatal necrotising enterocolitis: a systematic review and meta-analysis of observational studies. Arch Dis Child Fetal Neonatal Ed 2005;90:F147-F151

15 Cornet B, Gouyon JB, Binquet C, et al; Comité De Pilotage Du Réseau Périnatal De Bourgogne. [Using discharge abstracts as a tool to assess a regional perinatal network]. Rev Epidemiol Sante Publique 2001;49:583-593

16 Quantin C, Allaert FA, Gouyon B, Cohen O. Proposal for the creation of a European healthcare identifier. Stud Health Technol Inform 2005;116:949-954

17 Bin-Nun A, Bromiker R, Wilschanski M, et al. Oral probiotics prevent necrotizing enterocolitis in very low birth weight neonates. J Pediatr 2005;147:192-196

18 Lin HC, Su BH, Chen AC, et al. Oral probiotics reduce the incidence and severity of necrotizing enterocolitis in very low birth weight infants. Pediatrics 2005;115:1-4

19 Samanta M, Sarkar M, Ghosh P, Ghosh J, Sinha M, Chatterjee S. Prophylactic probiotics for prevention of necrotizing enterocolitis in very low birth weight newborns. J Trop Pediatr 2009;55:128-131

20 Lin $\mathrm{HC}$, Hsu CH, Chen HL, et al. Oral probiotics prevent necrotizing enterocolitis in very low birth weight preterm infants: a multicenter, randomized, controlled trial. Pediatrics 2008;122:693-700

21 Tarnow-Mordi WO, Wilkinson D, Trivedi A, Brok J. Probiotics reduce all-cause mortality and necrotizing enterocolitis: it is time to change practice. Pediatrics 2010;125:1068-1070 
22 Mihatsch WA. What is the power of evidence recommending routine probiotics for necrotizing enterocolitis prevention in preterm infants? Curr Opin Clin Nutr Metab Care 2011;14: 302-306

23 Deshpande GC, Rao SC, Keil AD, Patole SK. Evidence-based guidelines for use of probiotics in preterm neonates. BMC Med 2011; 9:92-110

24 Beattie LM, Hansen R, Barclay AR. Probiotics for preterm infants: confounding features warrant caution. Pediatrics 2010;126: e742-e743; author reply e743-e745

25 Indrio F, Riezzo G, Raimondi F, Bisceglia M, Cavallo L, Francavilla R. Effect of probiotic and probiotic on gastrointestinal motility in newborns. J Physiol Pharmacol 2009;60:S27-S31

26 Indrio F, Riezzo G, Raimondi F, Bisceglia M, Cavallo L, Francavilla R. The effects of probiotics on feeding tolerance, bowel habits, and gastrointestinal motility in preterm newborns. J Pediatr 2008; 152:801-806
27 Satoh Y, Shinohara K, Umezaki H, et al. Bifidobacteria prevents necrotising enterocolitis and infection. Int $\mathrm{J}$ Probiot Prebiot 2007;2:149-154

28 Maayan-Metzger A, Ghanem N, Mazkereth R, Kuint J. Characteristics of neonates with isolated rectal bleeding. Arch Dis Child Fetal Neonatal Ed 2004;89:F68-F70

29 Baldassarre ME, Laforgia N, Fanelli M, Laneve A, Grosso R, Lifschitz C. Lactobacillus $G G$ improves recovery in infants with blood in the stools and presumptive allergic colitis compared with extensively hydrolyzed formula alone. J Pediatr 2010;156:397-401

30 Manzoni P, Mostert M, Leonessa ML, et al. Oral supplementation with Lactobacillus casei subspecies rhamnosus prevents enteric colonization by Candida species in preterm neonates: a randomized study. Clin Infect Dis 2006;42:1735-1742

31 Brand S, Reickenecker HC. An enhanced barrier is a better defence: effects of probiotics on intestinal barrier function. Inflamm Bowel Dis 2002;8:67-69 